\title{
ПРОГРАММНЫЙ АНАЛИЗ ТИПИЧНЫХ АТАК НА АЛГОРИТМ DES
}

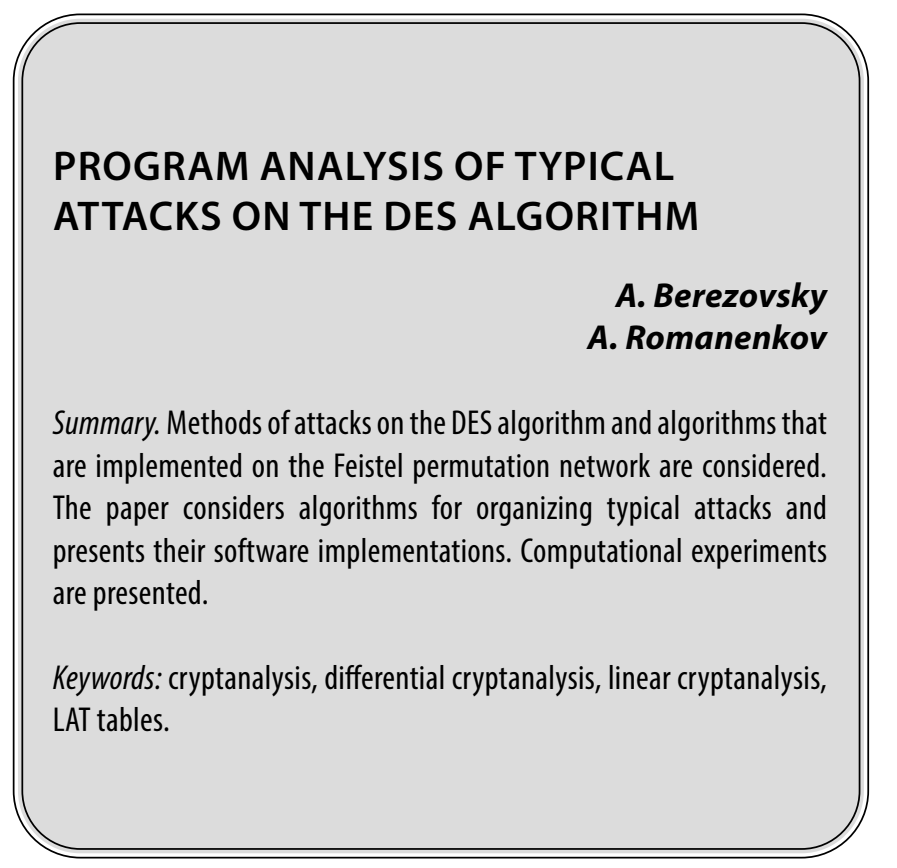

\section{Введение}

A лгоритм DES (Data Encryption Standard) - это один из распространённых алгоритмов. На протяжении нескольких лет (1977-1980 г.) был национальным стандартом шифрования США. На замену этого алгоритма пришёл новый алгоритм шифрования данных Rijndail, который является стандартом шифрования США и по сей день. На основе DES предложен новый алгоритм, который называется 3DES, разработанный в 1978 году [1].

При взломе DES в основном использовались следующие атаки:

1. Метод полного перебора. Требует одну известную пару шифрованного и расшифрованного текста, его выполнение требует около $2^{55}$ шагов.

2. Дифференциальный криптоанализ. Первую такую атаку на DES заявили Бихам и Шамир. Эта атака требует шифрования $2^{47}$ открытых текстов, выбранных нападающим, и для её выполнения нужны примерно $2^{47}$ шагов. Но оказалась не практичной.

3. Линейный криптоанализ. Разработан Matsui. Этот метод позволяет восстановить ключ DES с помощью анализа $2^{43}$ известных открытых текстов. Первый экспериментальный криптоанализ DES, основанный на открытии Matsui, был успешно выполнен в течение 50 дней
Березовский Александр Дмитриевич

Московский авиачионный институт

(национальный исследовательский университет),

Москва

sashulber@mail.ru

Романенков Александр Михайлович

К.т.н., доцент, Московский авиационный институт (национальный исследовательский университет); с.н.с., ФИЦ «Информатика и управление» Российской Академии Наук, Москва romanaleks@gmail.com

Аннотация. Рассмотрены методы атак на алгоритм DES и алгоритмы, которые реализованы на подстановочно-перестановочной сети Файстеля. В работе рассмотрены алгоритмы организации типичных атак и представлены их программные реализации. Приведены вычислительные эксперименты.

Ключевые слова: криптоанализ, дифференциальный криптоанализ, линейный криптоанализ, LAT таблицы.

на автоматизированных рабочих местах $12 \mathrm{HP}$ 9735 [2].

4. Атака встречей по середине. Основной принцип атаки заключается в разделении раундов на верхнюю и нижнюю части. Главное условие, от которого отталкивается атакующий - это равенство по середине разделённых раундов [8].

\section{Описание алгоритма DES}

DES шифрует блоки длиною 64 бит. Алгоритм является типичной сетью Фейстеля с 16 раундами. В каждом раунде используется свой раундовый ключ, которые генерируются из основного ключа, вводимого пользователем. В алгоритме DES16 раундовых ключей, которые имеет длину 48 бит [2].

Основная функция шифрования:

- расширяющая перестановка $E$,

- сложение по модулю 2 с раундовым ключом $k_{i}$.

- преобразование $S$ с помощью восьми $S$ блоков $\left(S_{1}, S_{2}, \ldots, S_{8}\right)$.

- перестановка $P$.

Механизм работы сети Фейстеля заключается в преобразовании блоков, которые разбиваются на две части (левая и правая). Правая $R_{i}$ копируется в левую часть следующего блока $L_{i+1}$, а $L_{i}$ складывается по модулю 2 с $R_{i}$ прошедшую заданные преобразования ра- 


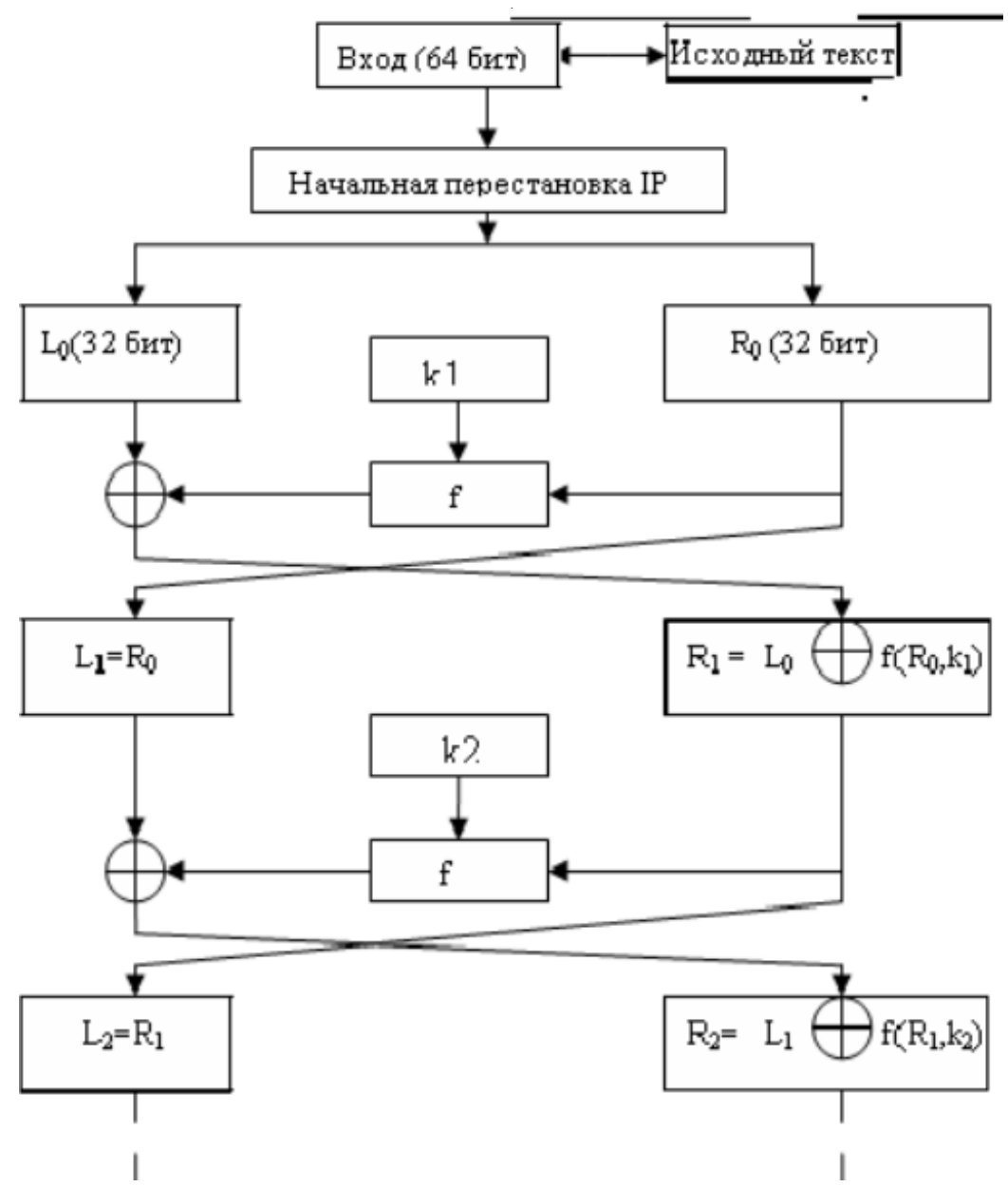

Рис. 1. Схема двух раундов DES

унда $f$ и присваивается правой части следующего блока $R_{i+l}$. Таким образом циклично преобразуются несколько блоков [3].

\section{Криптоднализ}

\section{Атака грубой силой}

Всеми известная атака «брутфорс» или по-другому атака перебором ключа. Данная атака не уместна к алгоритмам шифрования подобным шифру Вермана. Так как все перебираемые ключи также перебирают всё возможные открытые тексты [4].

Основная суть атаки заключается в последовательном переборе ключа. Нужно чтобы подбираемый ключ совпал с секретным. Об этом должно сообщить появление определённого сигнала. Сигнал зависит от условий в которых и при которых проходит атака.

Атака грубой силой в своём подавляющем большинстве гарантирует, что данный шифр будет взломан, за некоторое количество времени [4]. Была создана машина DES Cracker организацией Electronic Frontier Foundation, которой удалось взломать алгоритм DES меньше чем за неделю.

Атаке грубой силой невозможно противостоять, не расширяя количество секретных бит. Как и поступили создатели 3DES. Ключ в данном алгоритме составляет 168 бит (эффективные лишь 112 бит), что в разы больше, чем 56 бит обычного DES [1].

\section{Атака линейным анализом}

Суть метода состоит в нахождении линейной зависимости начального текста, шифротекста и ключа. После нахождения зависимостей выбирается наиболее эффективная и подбираются оставшиеся биты прямым перебором.

Прежде чем приступить к DES, рассмотрим подстановочно-перестановочную сеть ( $S P$-cemb). Пусть $x, y-$ это двоичные вектора размерностью $n$. Тогда скалярное произведение этих векторов определим формулой: 


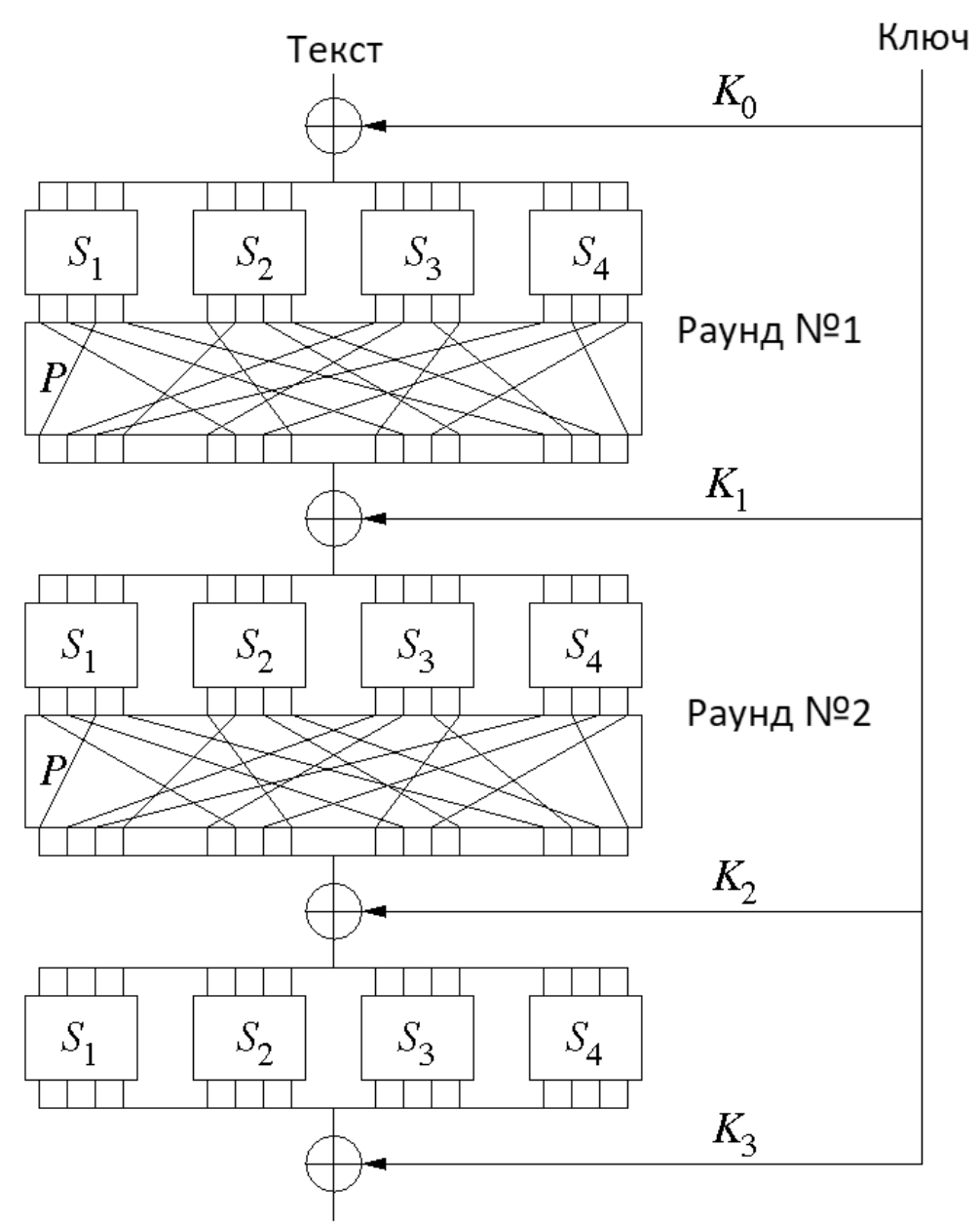

Шифротекст

Рис. 2. Схема SP-сети с 4-мя S блоками

$$
<x, y>=x_{1} \cdot y_{1} \oplus \ldots \oplus x_{n} \cdot y_{n}
$$

где операция (·) - это поразрядная конъюнкция, a $(\oplus)$ — это поразрядное исключающие «или» (XOR).

Пусть, далее, Р - открытый текст, С - шифротекст, $\mathrm{K}$ - ключ, $\epsilon$ - преобладание линейного соотношения, $\alpha, \beta, \gamma$ - выбранные двоичные векторы, которые будем называть масками. Линейным приближением шифра будем называть:

$$
<P, \alpha>\oplus<C, \beta>=\langle K, \gamma>
$$

который будет выполняться с вероятностью $1 / 2+\epsilon$. Чем больше $|\epsilon|$, тем успешнее будет криптоанализ.

Для наглядности рассмотрим раунд № 1, в котором S блоки $S_{1}=S_{2}=S_{3}=S_{4}$. Значения для всех S возьмём равные $(0,1,13,12,11,10,9,8,7,6,5,4,3,2,14,15)$. Рассмотрим все возможные приближения $\mathrm{S}$ блока $\langle a, x\rangle$ и $<b, y\rangle$, где $a$ и $b \in Z_{2}^{4}$. Теперь рассмотрим линейное приближение, например:

$$
X_{1} \oplus X_{2}=Y_{1} \oplus Y_{2} \oplus Y_{3}
$$

то есть здесь масками являются вектора $a=(1100)=12, b=(1110)=14$. В итоге можно получить таблицу всех возможных вариаций входных данных и выходных. В этой таблице -столбцы - это все возможные вариации левой части линейного приближения, $Y$-столбцы - это все возможные вариации правой части линейного приближения (рис. 3).

При анализе значений в предпоследнем и последнем столбцах, заметим, что равенство выполняется в 10-ти строчках из 16-ти. Получается, что равенство выполняется с вероятностью в

$$
\epsilon_{1}=\frac{10}{16}-\frac{1}{2}=\frac{2}{16} \text {. }
$$




\begin{tabular}{r|r|r|r|r|r|r|r|r|r|r|}
$\mathrm{X} 1$ & $\mathrm{X} 2$ & $\mathrm{X} 3$ & $\mathrm{X} 4$ & $\mathrm{Y} 1$ & $\mathrm{Y} 2$ & $\mathrm{Y} 3$ & $\mathrm{Y} 4$ & $\mathrm{X} 1+\mathrm{X} 2$ & $\mathrm{Y} 1+\mathrm{Y} 2+\mathrm{Y}$ \\
\hline 0 & 0 & 0 & 0 & 0 & 0 & 0 & 0 & 0 & 0 \\
\hline 0 & 0 & 0 & 1 & 0 & 0 & 0 & 1 & 0 & 0 \\
\hline 0 & 0 & 1 & 0 & 1 & 1 & 0 & 1 & 0 & 0 \\
\hline 0 & 0 & 1 & 1 & 1 & 1 & 0 & 0 & 0 & 0 \\
\hline 0 & 1 & 0 & 0 & 1 & 0 & 1 & 1 & 1 & 0 \\
\hline 0 & 1 & 0 & 1 & 1 & 0 & 1 & 0 & 1 & 0 \\
\hline 0 & 1 & 1 & 0 & 1 & 0 & 0 & 1 & 1 & 1 \\
\hline 0 & 1 & 1 & 1 & 1 & 0 & 0 & 0 & 1 & 1 \\
\hline 1 & 0 & 0 & 0 & 0 & 1 & 1 & 1 & 1 & 0 \\
\hline 1 & 0 & 0 & 1 & 0 & 1 & 1 & 0 & 1 & 0 \\
\hline 1 & 0 & 1 & 0 & 0 & 1 & 0 & 1 & 1 & 1 \\
\hline 1 & 0 & 1 & 1 & 0 & 1 & 0 & 0 & 1 & 1 \\
\hline 1 & 1 & 0 & 0 & 0 & 0 & 1 & 1 & 0 & 1 \\
\hline 1 & 1 & 0 & 1 & 0 & 0 & 1 & 0 & 0 & 0 \\
\hline 1 & 1 & 1 & 0 & 1 & 1 & 1 & 0 & 0 & 0 \\
\hline 1 & 1 & 1 & 1 & 1 & 1 & 1 & 1 & 0 & 0 \\
\hline
\end{tabular}

Рис. 3. Таблица истинности для линейного приближения

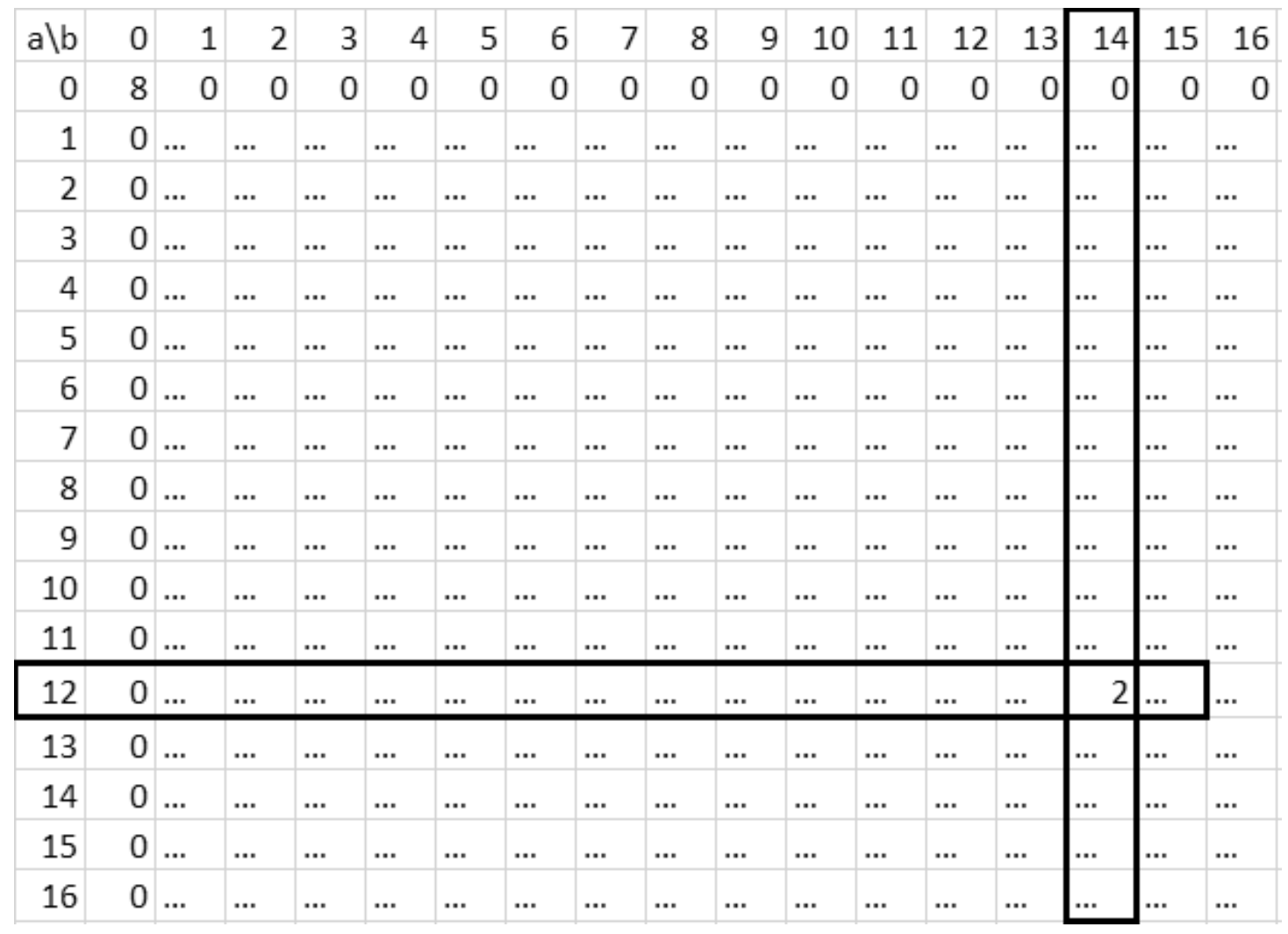

Рис. 4. LAT таблица 


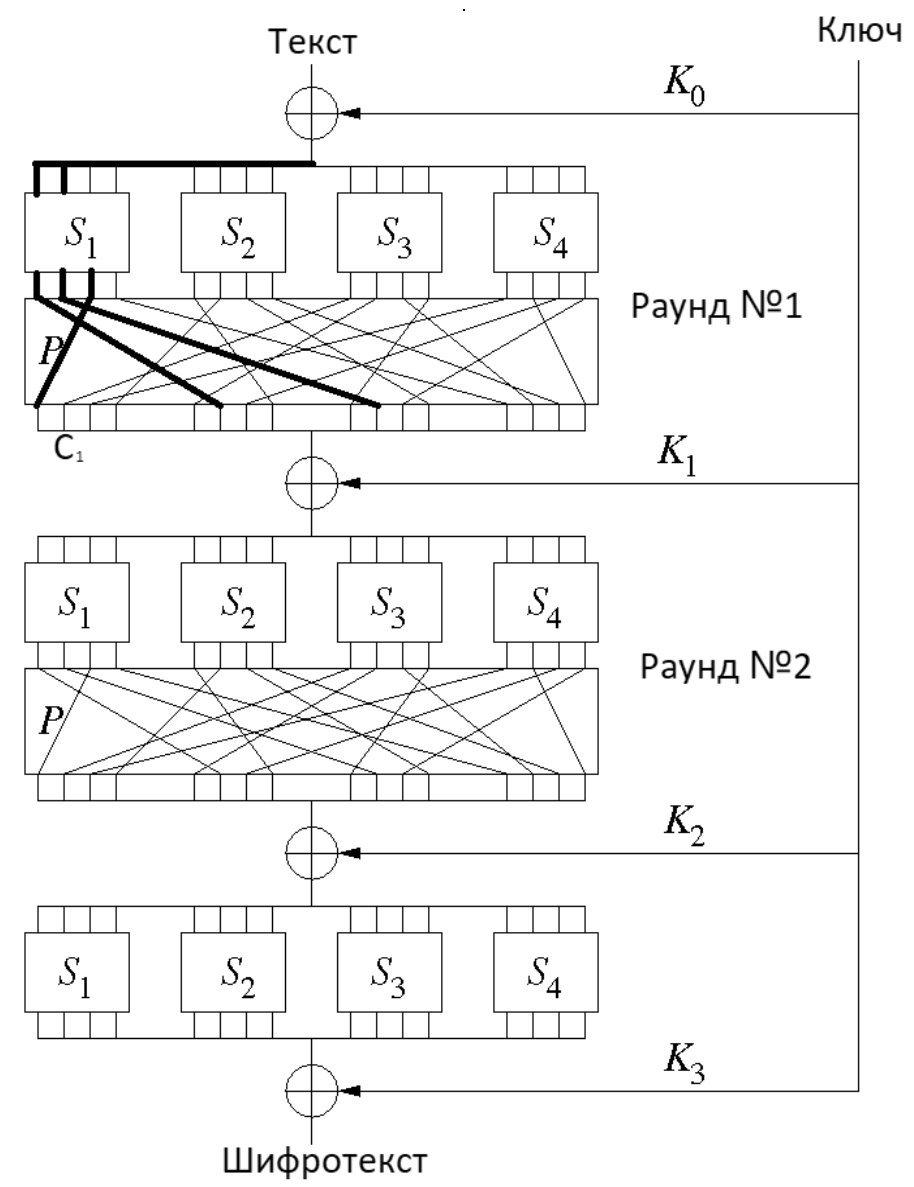

Рис. 5. Схема SP-сети с 4-мя S блоками

Далее строится так называемая LAT таблица для S-блока (таблица линейного приближения). В общем случае для $S$-блока это $n \times n$ таблица будет размера $2^{n}$ на $2^{n}$, где строки - это векторы $a$, а столбцы это векторы $b$ (рис. 4), где значения $-8,8,0$ не будут интересовать. Эффективнее брать 4,-4, 6,-6 для дальнейших вычислений. Знак перед значением из ячейки не важен, так как при отрицательном $\epsilon$ в линейном приближении (в правой части) добавляется $\oplus 1$.

Рассмотрим структуру шифра 1-го раунда (рис. 5).

Как видно при входе получается $P[1,2]$ (для 1-го S блока), $K_{0}[1,2]$, а на выходе $C_{1}[1,6,10]$. Далее на вход в следующий раунд подаётся $C_{1}[1,6,10]$. В итоге выходит линейное приближение для 1-го раунда:

$$
P[1,2] \oplus K_{0}[1,2]=C_{1}[1,6,10]
$$

это приближение только для одного раунда. Таким образом итерируются эти операции на всех раундах.

Следующим шагом будет рис. 6, то есть система разобьётся на

$$
\begin{aligned}
& C_{1}[1] \oplus K_{1}[1]=C_{2}\left[n_{1}, m_{1}\right] \\
& C_{1}[6] \oplus K_{1}[6]=C_{2}\left[n_{2}, m_{2}\right] \\
& C_{1}[10] \oplus K_{1}[10]=C_{2}\left[n_{3}, m_{3}\right]
\end{aligned}
$$

где $n, m$ нужно посчитать тем же самым способом. Далее формируется линейное приближение уже для 2-го раунда:

$P[1,2] \oplus K_{0}[1,2] \oplus K_{1}[1,6,10]=$

$=C_{2}\left[n_{1}, m_{1}, n_{2}, m_{2}, n_{3}, m_{3}\right]$

и результирующая вероятность, с которой выполняется данное неравенство ищется с помощью леммы Мацуи «о набегании знаков»:

Лемма Мацуи [5]. Пусть $X_{i,}$ где - независимые случайные величины, принимающие значения из $Z_{2}$. $\Pi$ Псmb

$$
\begin{aligned}
& P\left\{X_{i}=0\right\}=\frac{1}{2}+\epsilon_{i} \\
& \text { где } 0 \leq \epsilon_{i} \leq \frac{1}{2} .
\end{aligned}
$$




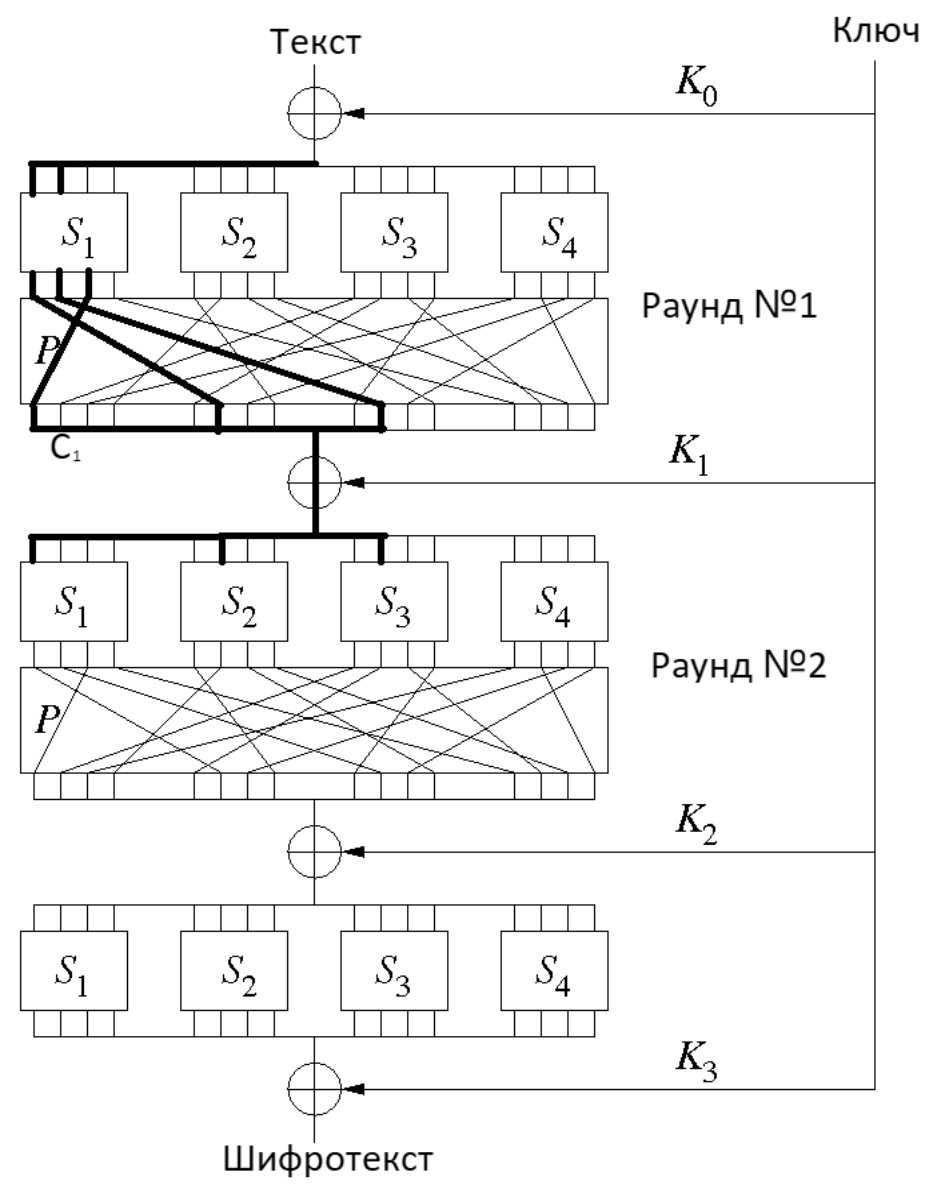

Рис. 6. Схема SP-сети с 4-мя S блоками

Тогда случайная величина $X_{1} \oplus X_{2} \oplus \ldots \oplus X_{n}$ принимает значение 0 с вероятностью

$\frac{1}{2}+\epsilon$, где $\epsilon=2^{n-1} \prod_{i=1}^{n} \epsilon_{i}$.

Вероятность для 2-го раунда будет равна

$\frac{1}{2}+2^{3}\left(\frac{2}{16}\right)\left(\frac{t}{p}\right)$, где $\frac{t}{p}-$

это вероятность выполнения равенства во втором раунде, где $t$ - числитель, который взят из LAT-таблицы. И завершающий этап алгоритма имеет несколько своих вариаций. Рассмотрим следующую:

После нахождения окончательного линейного соотношения (назовём его $P C K(2)$, а также вероятности с которой оно выполняется

$$
p=\frac{1}{2}+\epsilon
$$

для каждой пары $P, C$ вычисляется значение левой части соотношения $P C K$. Пусть $N_{0}, N_{1}$ будут количество пар статистики, для которых левая часть соотношения равна 0 и 1 (очевидно $N_{0}+N_{1}=N$ ).
Положим $\langle K, \gamma\rangle=0$, если $\left(N_{0}-N_{1}\right) * \epsilon>0$ и $\langle K, \gamma\rangle=1$, если $\left(N_{0}-N_{1}\right) \cdot \epsilon \leq 0$. И с учётом этого условия подбирается ключ грубой силой [5].

Теперь отобразим те же действия только с учётом этапов шифрования DES:

Первая проблема, с которой можно столкнутся это то, что в начале идёт не объединение с ключом, а преобразование правой части текста матрицей расширения Е. Поэтому можно сказать, что вместо R получается $E(R)$, где $E$ - это преобразование матрицей расширения и $R$ - это правая часть текста P, т.е. $R \rightarrow E(R)$.

Далее будет ровно такая же процедура сложения по модулю 2 с раундовым ключом.

Вторая проблема, с которой можно столкнутся, это необычный вход на S блок:

Входят 6 битов, а выходит всего 4, то есть все возможные вариации входа будут не 4 столбца, а 6 . Но для выходов все равно будет 4 столбца. Строк в этом случае будет 64 (это все возможные вариации выхода из S блока). 


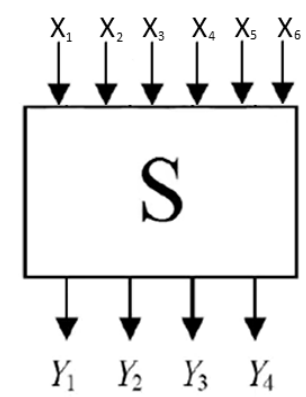

Рис. 7. S блок в алгоритме DES

\begin{tabular}{|c|c|c|c|c|c|c|c|c|c|c|c|c|c|c|}
\hline 3 & 4 & 5 & & 6 & 7 & 8 & 9 & 10 & 11 & 12 & 13 & 14 & 15 & \\
\hline-2 & 0 & -2 & 4 & & -6 & 2 & 4 & 2 & 4 & -2 & -8 & 10 & -4 & $\wedge$ \\
\hline 2 & 0 & 2 & -4 & 4 & 6 & -6 & 0 & 2 & -8 & -2 & -4 & 10 & 0 & \\
\hline 4 & 2 & -4 & 0 & & 2 & 2 & 0 & -4 & -6 & 0 & -2 & -2 & 4 & \\
\hline 8 & -2 & 8 & 0 & & 2 & -2 & 4 & 4 & -6 & 8 & -2 & 2 & 0 & \\
\hline 0 & 2 & 2 & 6 & & -10 & -2 & 2 & 2 & -2 & 0 & 4 & 8 & 20 & \\
\hline-8 & -2 & 2 & -2 & 2 & 2 & 2 & 2 & 2 & -6 & 0 & 0 & -4 & 4 & \\
\hline-2 & 0 & 4 & -2 & 2 & -6 & -2 & 6 & -4 & 4 & -2 & 6 & -8 & 0 & \\
\hline 6 & 0 & -4 & -2 & 2 & 2 & 6 & 6 & -4 & -4 & -2 & -2 & 0 & 0 & \\
\hline 0 & -4 & 4 & 0 & & -4 & 4 & -4 & 0 & 4 & 0 & 4 & 0 & 0 & \\
\hline-8 & 0 & 4 & 8 & & 0 & -4 & 0 & -4 & -4 & 4 & -4 & 8 & -4 & $\checkmark$ \\
\hline Выб & я $\mathrm{Cl}$ & & 16 & Can & e эфф & $\mathrm{BHHC}$ & Іачен & стро & 20 & Само & $\phi \phi е к$ & Hoe $3 \mathrm{r}$ & ение в таблице: & 20 \\
\hline
\end{tabular}

Рис. 8. LAT таблица 5-го S блока

Потом выходные биты просто складываются по модулю 2 с другой 32-х битной частью. Т.е. в итогом соотношении пропадает $\mathrm{P}$ и $\mathrm{C}$, а появляются $\mathrm{R}$, F, L. Учитывается, что $R_{i-1}$ становится $\left.L_{i}\right\}$, а также $F\left(R_{i-1}, K_{i}\right)[m, \ldots] \oplus L_{i-1}$, становится $R_{i}$. Линейное приближение будет иметь вид:

$$
R_{i-1}[n, \ldots] \oplus F\left(R_{i-1}, K_{i}\right)[m, \ldots]=K_{i}[t, \ldots]
$$

для 1-го раунда.

Известное линейное соотношение эффективной атаки на DES:

Для нахождения соотношения Мацуи использовал следующие приближения S блоков:

$$
\begin{aligned}
& S_{5}: x_{2}=y_{1} \oplus y_{2} \oplus y_{3} \\
& S_{1}: x_{2}=y_{1} \oplus 1 \\
& S_{5}: x_{2}=y_{1} \oplus y_{2} \oplus y_{3} \oplus y_{4} \oplus 1
\end{aligned}
$$

и в итоге получает вероятности

$\frac{42}{64}, \frac{34}{64}, \frac{52}{64}$
Тем самым прийдя к результату:

$\epsilon_{i}=\frac{52}{64}-\frac{1}{2}=\frac{20}{64}$ благодаря слабости $S \_5$ блока.

Мацуи выстроил специальную схему приближения, для нахождения итогового линейного соотношения:

$R_{1}[8,14,25] \oplus L_{15}[17] R_{1}[8,14,25,3]=$

$=K_{3}[26] \oplus K_{4}[4] \oplus K_{5}[26] \oplus K_{7}[26] \oplus$

$\oplus K_{8}[4] \oplus K_{9}[26] \oplus K_{11}[26] \oplus K_{12}[4] \oplus$

$\oplus K_{13}[26] \oplus K_{15}[26]$

и получил итоговое преобладание $\epsilon=0,00000057$, что практически полностью уничтожило криптостойкость шифра алгоритмом DES [6]. Чтобы убедиться, в 5-м S блоке действительно есть слабость, воспользуемся программой (написанной на С\#) и убедимся, что такое значение существует (рис. 8).

Действительно, при значениях $a=010000$ и $b=1111$ получается самый эффективный проход через $S_{5}$ блок.

Рассмотрим код, который может вызвать затруднения в реализации. Случай, когда подготавливается правая часть линейного приближения (а точнее все возможные варианты), это $Y_{1}, Y_{2}, Y_{3}, Y_{4}$. По сути, просто 
Таблица S блока:

\begin{tabular}{|c|c|c|c|c|c|c|c|c|c|c|c|c|c|c|c|}
\hline 14 & 4 & 13 & 1 & 2 & 15 & 11 & 8 & 3 & 10 & 6 & 12 & 5 & 9 & 0 & 7 \\
\hline 0 & 15 & 7 & 4 & 14 & 2 & 13 & 1 & 10 & 6 & 12 & 11 & 9 & 5 & 3 & 8 \\
\hline 4 & 1 & 14 & 8 & 13 & 6 & 2 & 11 & 15 & 12 & 9 & 7 & 3 & 10 & 5 & 0 \\
\hline 15 & 12 & 8 & 2 & 4 & 9 & 1 & 7 & 5 & 11 & 3 & 14 & 10 & 0 & 6 & 13 \\
\hline
\end{tabular}

\begin{tabular}{|c|c|c|c|c|c|c|c|c|c|c|c|c|c|c|c|c|c|}
\hline & 1 & 2 & 3 & 4 & 5 & & 6 & 7 & 8 & 9 & 10 & 11 & 12 & 13 & 14 & 15 & \\
\hline 0 & 2 & 0 & 2 & 0 & 6 & & 0 & 6 & -2 & 0 & 2 & -4 & 6 & -4 & 2 & 0 & $\wedge$ \\
\hline 0 & -2 & -4 & 2 & 0 & -6 & & 4 & 6 & -6 & 0 & 2 & -8 & -6 & -4 & 2 & 4 & \\
\hline 0 & -4 & -2 & -2 & 2 & -6 & & 0 & 4 & 0 & -4 & -2 & -2 & 2 & 2 & 0 & -4 & \\
\hline 0 & -4 & -2 & -2 & -2 & -2 & & 4 & 0 & 0 & -4 & -2 & -2 & 6 & -2 & -4 & 0 & \\
\hline 0 & 0 & 2 & -6 & -2 & -2 & & -4 & 4 & -2 & -6 & 0 & 4 & 0 & 4 & 6 & 18 & \\
\hline 0 & -4 & 2 & -2 & -2 & 2 & & -4 & 0 & 2 & 2 & -4 & -4 & -4 & -4 & 10 & -6 & \\
\hline 0 & -6 & -4 & 2 & 0 & 2 & & 0 & 6 & 4 & 2 & 4 & 6 & 0 & 6 & 4 & -10 & \\
\hline 0 & 2 & 0 & -2 & 4 & 6 & & 8 & -2 & -4 & 2 & 0 & -6 & 4 & -6 & 12 & -2 & \\
\hline 0 & 2 & -8 & 6 & 0 & -2 & & 4 & -2 & 4 & 6 & -4 & 2 & 4 & 2 & 0 & 2 & \\
\hline 0 & -10 & 4 & 6 & -4 & -2 & & -4 & -6 & 0 & -2 & -4 & -2 & 4 & -2 & 4 & 2 & $\checkmark$ \\
\hline S блок & S1 & $\checkmark$ & Выбр & ая ст & & 17 & & е эфс & ИВНС & аче & строч & 10 & Cam & $\phi \phi е к ~$ & Hoe $3 \mathrm{r}$ & ение в таблице: & 18 \\
\hline
\end{tabular}

Рис. 9. LAT таблица 1-го S блока

достаются из выбранной матрицы S все возможные варианты, которые заданы константами, где $\left(y_{1} y_{2} y_{3} y_{4}\right)$ это значения от 0b0000 до 0b1111:

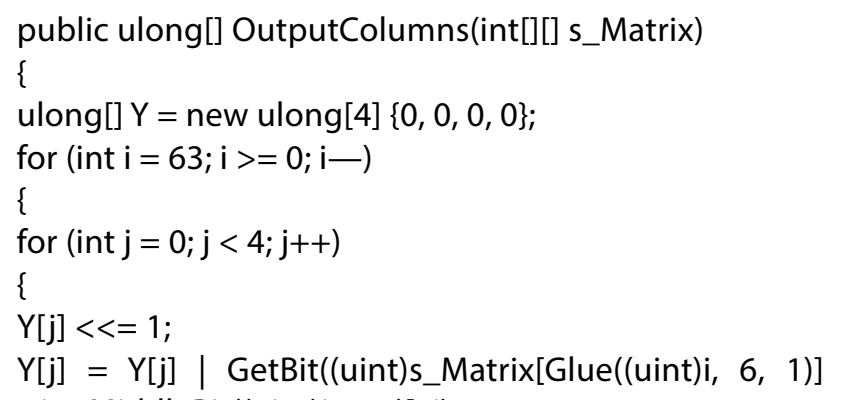
[CuttingMiddleBit((uint)i, 6, 1)], j);

\}

\}

return $Y$;

\}

// GetBit - ставит n-ый бит на 0-й порядок

// Glue - склеивает левую и правую части битового массива

// CuttingMiddleBit - вырезает левую и правую части битового массива

С использованием вычислительного эксперимента можно убедиться, что $S_{5}$ является самый уязвимый $\mathrm{S}$ блок среди всех 8-ми.

Пример таблицы для $S_{1}$ блока - рис. 9.

Одной из главных функций является функция вычисления линейного преобразования правой и левой частей (по таблице истинности). Реализация для правой части выглядит следующим образом: public ulong LinearApproximation_RightPart(uint rightPart,

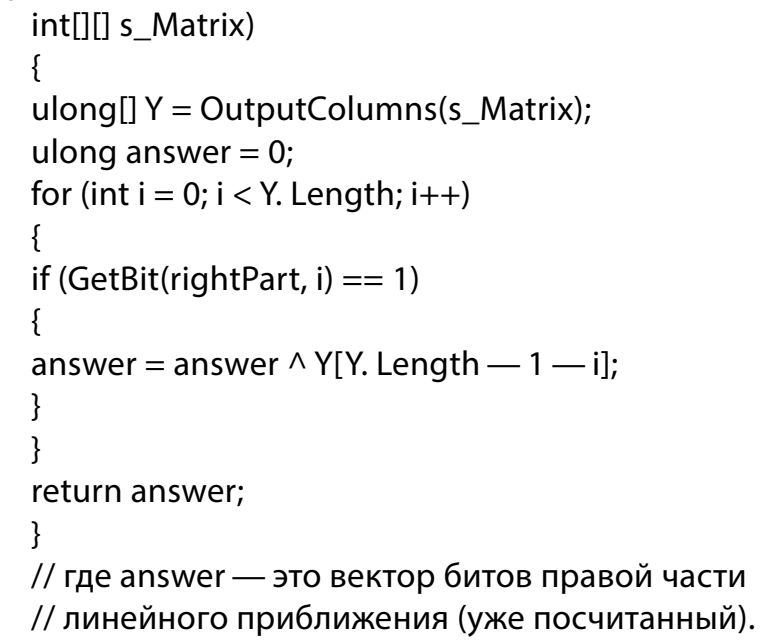

После получения двух векторов левой и правой части линейного преобразования, значения векторов сравниваются. Количество совпавших значений сохраняется в $n$. Всего имеется $m=64$ значений. Подсчитывается значение для одной ячейки в LAT таблице

$$
\left(\frac{n}{m}\right) \text {. }
$$

Такие действия выполняются для всех ячеек LAT таблицы. Для определения самого большого значения в таблице (исключая 32), воспользуемся функцией FindEffectiveValuePerTable:

public static int FindEffectiveValuePerTable(int[][] LAT_table) 
Входные данные и взаимодействие

10-ая система:

13312312340512312312

2-ая система:

\section{0}

\section{Начать}

След раунд

Сбросить

Результаты и вычисления

Вероятность приближения $(\mathrm{p})=$

$(-4 / 64)^{\star}(-4 / 64)^{\star}(0 / 64)^{\star}(8 / 64)^{\star}(-2 / 64)^{\star}(-8 / 64)^{\star}(4 / 64) *(0 / 64)$

$\star 128+0.5=0,5$

Линейное приближение:

$\mathrm{P}[0,2,3,4,8,10,11,12,13,14,16,17,19,21,23$,

$24,28,30,32,33,38,39,41,44,47,52,54,55,56$,

$57,58,59,60]+K_{-} 0[2,3,10,11,12,13,15,20,23$,

$25,32,34,35,36,37,38,39,40,41,42,43,441$

$\mathrm{C}[0,1,6,7,9,12,15,20,22,23,24,25,26,27,28$, $=\quad 32,35,40,41,43,44,46,47,50,51,52,53,54,57$, 59]

Рис. 10. Вычисление линейной зависимости

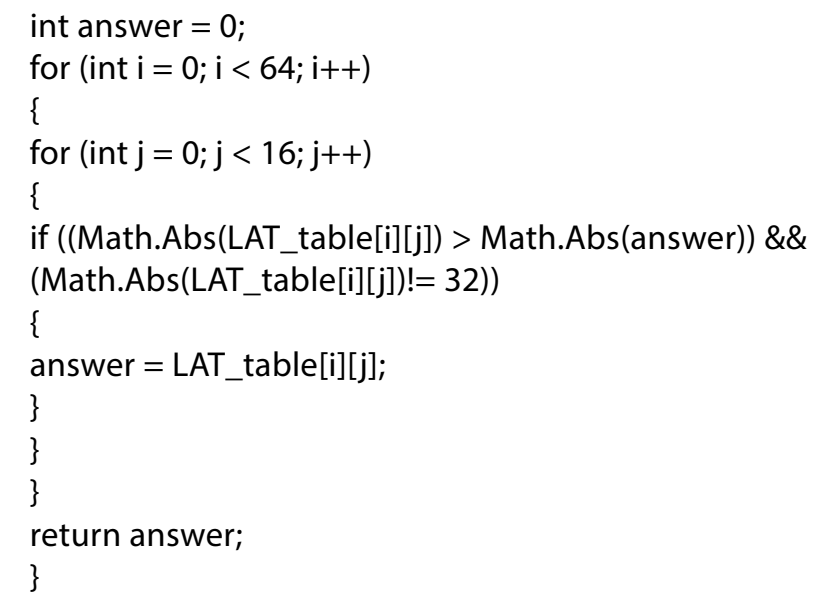

Таким образом можно наглядно увидеть все возможные уязвимости в S блоках. Чтобы вычислить линейную зависимость входного текста и выходного через линейное приближение можно обратиться к другому программному средству. В этом приложении вводится исходный текст и выполняется трассировка по раундам, с вычислением линейного приближения и вероятности линейного приближения. Не учитываются IP перестановки, так как программа работает непосредственно с переходами между раундами DES. Эти перестановки обратимы. Поэтому узнав информацию о входном раундовом тексте легко можно применить обратное IP преобразование, чтобы найти входной первоначальный текст (рис. 10).

В вероятности приближения (р) два множителя являются нулевыми, поэтому линейное приближение не подходит.

Данная программа не разделяет входные и выходные данные на левую и правую части. Они сразу склеиваются, образуя входные и выходные данные нужного размера в 64 бита.

Рассмотрим главную функцию программы. Эта функция получает входной текст, помещает его в переменную tmp_P, далее в массив ключей_К сохраняются все биты tmp_P, а результаты всех преобразований сохраняются в переменную_С:

public ulong LinearApproximation_ForOneRound( ulong inputText64Bit)

\{

ulong tmp_P = inputText64Bit;

uint[] $\mathrm{L}=$ new uint[2];

uint[] $\mathrm{R}=$ new uint[2];

$\mathrm{L}[0]=$ (uint) (tmp_P $>>32)$;

$\mathrm{R}[0]=($ uint $)($ tmp_P \& $(($ ulong $) 1<<32)-1))$;

uint[][][] s_Matrixs $=$ new uint[8][][] \{ 


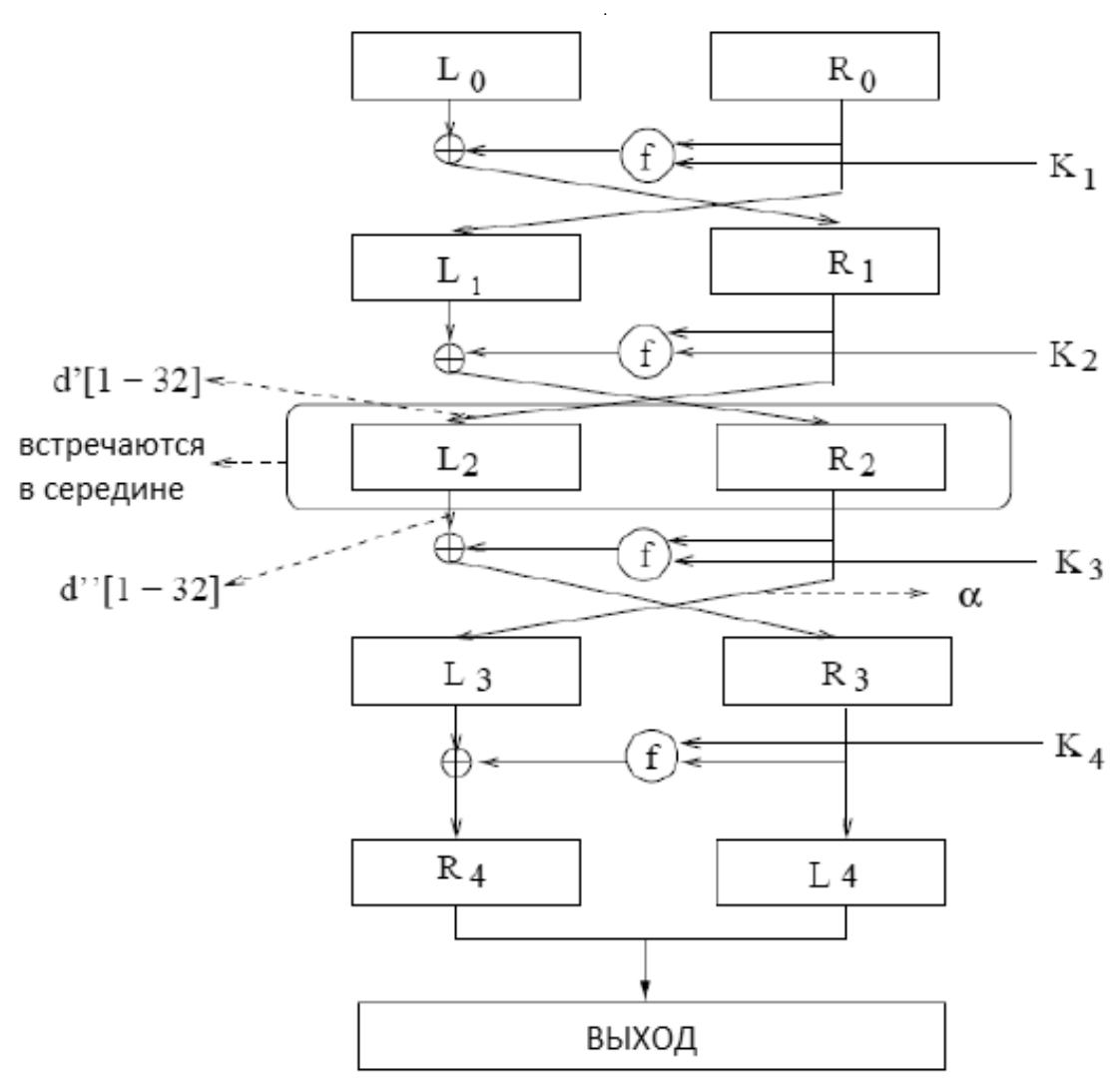

Рис. 11. Схема 4-х раундов DES

_S1,_S2,_S3,_S4,

_S5,_S6,_S7,_S8\};

tmp_P = BitsSwap(R[0], _E, 32);

$R[1]=L[0] \wedge P_{-}$Transformation(

S_Transformation(tmp_P, S_Matrixs));

$\mathrm{L}[1]=\mathrm{R}[0]$;

_K.Add(tmp_P); // массив ключей

_C $=(($ ulong $) \mathrm{L}[1]<<32) \mid \mathrm{R}[1]$;

return_C;

\}

Остальные функции взяты из программы по вычислению LAT таблицы.

Чтобы защититься от данной атаки нужно добавить новые S-блоки для алгоритма с учётом слабостей $S_{5}$ блока, а также увеличить размер ключа. Данная атака является самой эффективной для взлома алгоритма DES [7].

\section{Атака встречей по середине}

Суть атаки идёт по принципу «разделяй и властвуй», шифр делится на две части (верх и низ), после чего начинаем подбирать ключи с главным условием, что в середине интересующие биты из $L_{\text {middle }}$ или $R_{\text {middle }}$ [8].
1. Рассмотрим следующую простую схему двойного шифрования, которая вычисляет сообщение зашифрованного текста. С из текстового сообщения Р используя два ключа $K_{1}$, а также $K_{2}$ :

$$
\begin{aligned}
& C=E_{K_{2}}^{\prime}\left(E_{K_{1}}(P)\right) \\
& P=D_{K_{1}}^{\prime}\left(D_{K_{2}}(C)\right)
\end{aligned}
$$

Путём несложных вычислений можно вывести:

$$
\begin{aligned}
& C=E_{K_{2}}^{\prime}\left(E_{K_{1}}(P)\right) \\
& D_{K_{2}}^{\prime}(C)=D_{K_{2}}^{\prime}\left(E_{K_{2}}^{\prime}\left(E_{K_{1}}(P)\right)\right) \\
& D_{K_{2}}^{\prime}(C)=E_{K_{1}}(P)
\end{aligned}
$$

Это и есть основной вывод для использования атаки встречей по середине.

2. Рассмотрим более сложную схему. Пусть $M$ обозначает пространство сообщений, а $K$ обозначает пространство ключей:

Предположим, что $G_{k}, H_{k}: M \times K \rightarrow M-$ двухблочный шифр $F_{k}=G_{k} \circ H_{k}$. Злоумышленник пытается 
вывести K из заданной пары зашифрованного текста $c=F_{k}(p)$ пытаясь решить

$$
G_{-} k(p)=H_{k}^{-1}(c)
$$

Пусть $d^{\prime}[1-m]=G_{-} k(p), d^{\prime \prime}[1-m]=H_{k}^{-1}(c)$,

$G_{k}$ состоит из первых двух раундов DES

$H_{k}$ содержит раунды 3 и 4.

Рассмотрим $d^{\prime}[9-12]$ и $d^{\prime \prime}[9-12]$ (в скобках нумерация битов), достаточно угадать только 37 битов ключа. Если $d^{\prime}[9-12] !=d^{\prime \prime}[9-12]$, ключевое предположение не может быть правильным и отброшено.

Основное наблюдение заключается в том, что значения $d^{\prime}[9-12]$ и $d^{\prime \prime}[9-12]$ могут быть вычислены путём угадывания меньшего количества ключевых бит в обмен на угадывание внутренних битов (Е - функция расширения).

$$
\begin{aligned}
& d^{\prime}[9-12]=L_{-} 0[9-12] \oplus \\
& \oplus S_{-} 3\left[E\left(R_{-} 0\right)[13-18] \oplus K_{-} 1[13-18]\right] \\
& d^{\prime \prime}[9-12]=L_{-} 4[9-12] \oplus \\
& \oplus S_{-} 3\left[E\left(L_{-} 3\right)[13-18] \oplus K_{-} 3[13-18]\right]
\end{aligned}
$$

Замечание: Устанавливается зависимость битов [9-12] и [13-18] после функции расширения текста (и функции сжатия ключа). Так в 32-ух битах текста такие биты под номерами [9-12] будут находится между [13-18] в 48 расширенных битах текста.

$$
\begin{aligned}
& \text { Пусть } L_{3}=\left[\begin{array}{lll}
\alpha_{1} & \ldots . . \alpha_{32}
\end{array}\right] \text {, тогда } \\
& E\left(L_{3}\right)[13-18]=\left[\alpha_{17} \alpha_{1} \alpha_{15} \alpha_{23} \alpha_{26} \alpha_{5}\right] \text {. }
\end{aligned}
$$

Рассмотрим $\alpha_{17}$. можно было угадать все 37 предложенных ключевых битов, кроме 6 битов, составляющих $K_{4}[25-30]$. Для каждого предположения из 31 бита ключа злоумышленник пробует две возможности $\alpha_{17}$. Если для обоих значений равенство $d^{\prime}[9-12]=d^{\prime \prime}[9-12]$ не достигается, то предположение о 31 бите обязательно неверно [9].

Алгоритм хорошо себя показал при атаке на 3DES (нежели чем на DES), тем самым уменьшив эффективность его ключа с 168 до 112 бит [1]. Степень защиты атаки «встреча по середине» напрямую зависит от количества раундов. Увеличив количество раундов, увеличится и стойкость от таких атак.

\section{Атака дифференциальным анализом}

Основная идея данного метода состоит в изучении преобразований дифференцииалов (разностей) между шифруемыми значениями на различным этапах шифрования блоков. Дифференциал в данном случае - это результат сложения по модулю 2 некоторых параметров.

Характеристика - это пара дифференциалов, один из которых образован входными значениями некоторого преобразования, а второй - выходными значениями этого же преобразования. Дифференциал на входе преобразования также часто называют входной разностью, а дифференциал на выходе преобразования - выходной разностью. Входной дифференциал - это разность, которая подаётся на S блок. Выходной дифференциал это разность, которая получается после выхода с S блока.

Злоумышленник при использовании криптосистемы свободно оперирует с входными и выходными данными.

Непосредственная атака заключается в использовании заранее подготовленных текстах $M_{1}$ и $M_{2}$, где:

$$
\Delta M=M_{1} \oplus M_{2}
$$

и с помощью $\Delta M$ пытается определить каким должен быть дифференциал шифротекстов:

$$
\Delta C=C_{1} \oplus C_{2}
$$

Данный криптоанализ никогда не обеспечивает достоверное получение дифференциала шифротекстов. В основном это преобразование используется для вскрытия значительной части ключа с достаточной вероятностью, чтобы облегчить грубую атаку прямым перебором.

Предположим, что злоумышленник решил проверить дифференциал 0b10101010. Для этого выполняется генерация произвольного байта $X_{1}$, и вычисление:

$$
X_{2}=X_{1} \oplus 10101010
$$

Далее атакующий прогоняет $X_{1}$ и $X_{2}$ через функцию $S_{b o x l}$ и получает значения $Y_{1}$ и $Y_{2}$. Для каждой такой пары $X_{1}$ и $X_{2}$, дифференциал которых равен 0b10101010, атакующий в состоянии получить дифференциал $\Delta Y$. Анализируя полученные значения, атакующий выбирает такое значение $\Delta Y$, которое имеет большую вероятность возникновения.

Предположим, что из всех $m$ пар $X_{1}$ и $X_{2}$, в $n$ случаях $Y_{1} \oplus Y_{2}=101100$. Таким образом, вероятность того, что при заданном $\Delta X=0 b 10101010$, значение $\Delta Y=101100$, составляет $n / m$. Это в свою очередь означает, что при заданном 


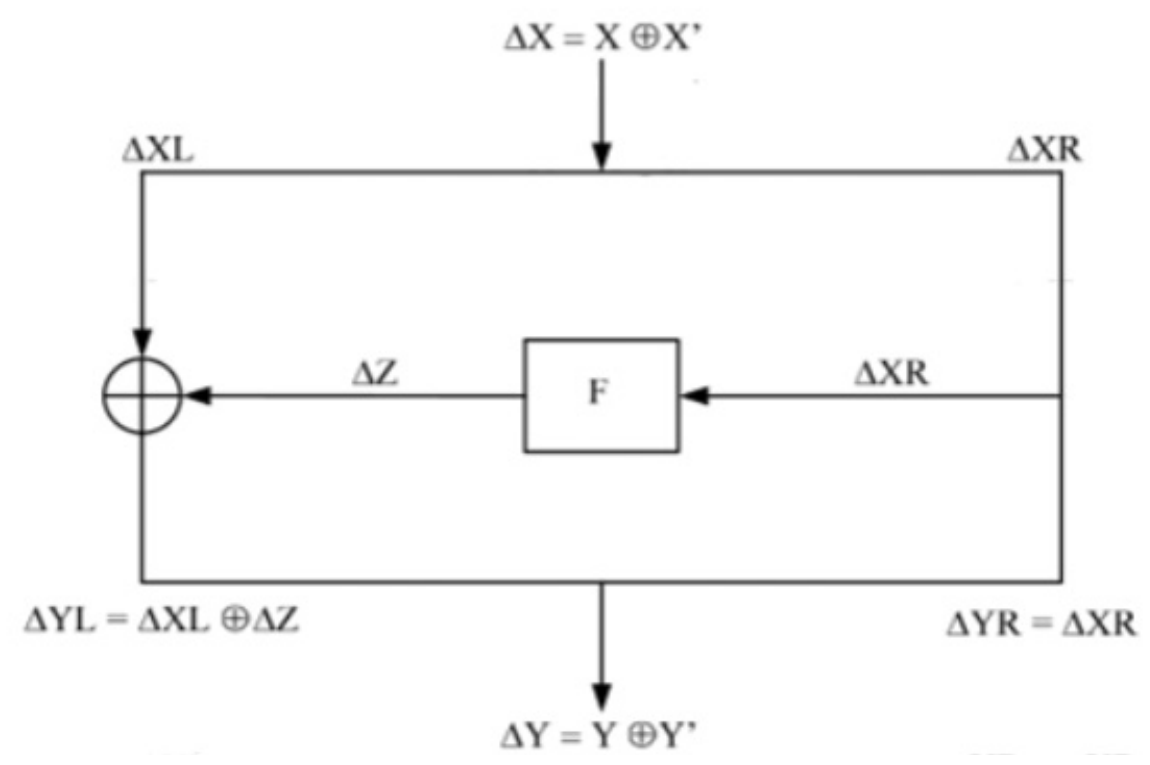

Рис. 12. Дифференциальный анализ 1-го раунда

$\Delta X=10101010$

с вероятностью

$P_{1}=\frac{n}{m}$

на вход второго раунда попадут два значения $U_{1}$ и $U_{2}$, такие что $\Delta U=101100$.

При следующих вычислениях число 101100 подаётся как входной дифференциал для следующего блока $S_{b o x 2}$. Проводятся те же вычисления для нахождения выходного дифференциала. Итоговая вероятность будет равна

$$
P=P_{1} \cdot P_{2}=\frac{n_{1}}{m_{1}} \cdot \frac{n_{2}}{m_{2}}
$$

Обладая этим фактом, атакующий генерирует несколько пар текстов таких, что $\Delta M=M_{1} ; M_{2} ; M_{3} ; \ldots$ где каждая $M_{n}=10101010$ - это и есть блок по 64 бита. Приступает к побайтовому подбору подключа. Вычисляется

$$
U_{-} 1=S_{b o x}\left(C_{1} \oplus \operatorname{key}[0]\right) U_{2}=S_{b o x}\left(C_{-} 2 \oplus \operatorname{key}[0]\right),
$$

где kеу[0] - это первый байт третьего раунда. Подобрав таким образом наиболее вероятный первый байт подключа, атакующий может перейти ко второму байту и действуя аналогичным образом вскрыть весь ключ третьего раунда [10].

Эта атака непрактична из-за чрезмерных требований к подбору данных и сложности организации атаки по выбранному открытому тексту (понадобится много памяти, а также относительно большая скорость обработки). Очевидная защита от таких атак заключается в увеличении размера раундового ключа, а также количества преобразований подобных S блокам [2].

\section{Зак^ючение}

В данной работе рассмотрены алгоритмы организации атак на алгоритм DES. Предложены программные решения, которые непосредственно реализуют эти алгоритмы. С помощью этих приложений выполнена визуализация этапов атаки линейным анализом, что позволяет самостоятельно определять уязвимости у классического алгоритма DES. Сгенерированы LAT таблицы для всех S блоков. А также были программно найдены исследованные Мацуи уязвимости.

Подробно проведён анализ 4-х распространённых атак на DES. Рассмотрены возможные варианты защиты от подобных атак.

\section{ЛИТЕРАТУРА}

1. 3DES // Википедия. Режим доступа: https://ru.wikipedia.org/wiki/Triple_DES (дата обращения: 24.09.2020).

2. DES // Википедия. Режим доступа: https://ru.wikipedia.org/wiki/DES (дата обращения: 25.09.2020).

3. Biryukov A., Christophe D. Data Encryption Standard (DES). In Encyclopedia of Cryptography and Security. Netherlands: 2011. Vol. 3. 691 p. 
4. Смарт Н. Криптография. М.: Техносфера, 2006. 528 c.

5. Matsui M. Linear cryptanalysis method for DES cipher // Computer \& Information Systems Laboratory. 1998. Pp. 1-12.

6. P. Patil, P. Narayankar, D.G. Narayan, S.M. Meena. A Comprehensive Evaluation of Cryptographic Algorithms: DES, 3DES, AES, RSA and Blowsh // Procedia Computer Science. 2016. Pp. 1-8.

7. Pascal J. On the Complexity of Matsui's Attack // Security and Cryptography Laboratory. 2001. Pp. 1-13.

8. Stephane M. Meet-in-the-Middle Attacks: Autoref. Dis. ... Doctor of Engineering Sciences: "Analytical". Thousand Oaks, California, U.S., 2010. 2 p.

9. Li J. Attack on DES: Autoref. Dis. ... Doctor of Engineering Sciences: "Analyst". California, 1985. 33 p.

10. Howard M. Linear and Differential Cryptanalysis: Autoref. Dis. ... Doctor of Engineering Sciences: "Cryptographer". St. John's, NL, Canada, 2002.33 p.

11. Grabbe J. The DES algorithm illustrated // Laissez Faire City Times. 1992. Pp. 1-13.

(c) Березовский Александр Дмитриевич ( sashulber@mail.ru ), Романенков Александр Михайлович ( romanaleks@gmail.com ).

Журнал «Современная наука: актуальные проблемы теории и практики»

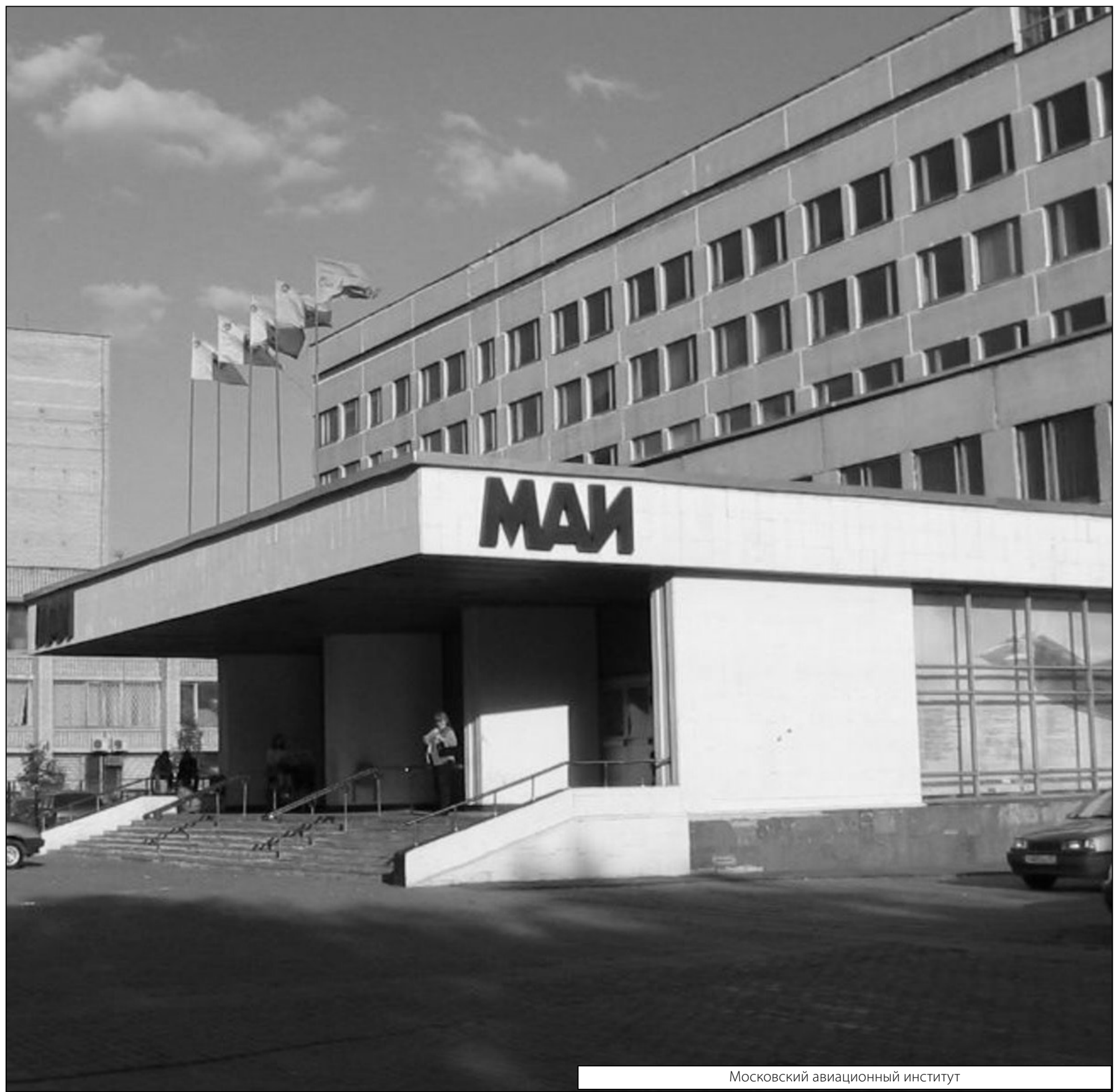

